\title{
Harmonic dynamic loading response of reinforced concrete column
}

\author{
Saba Sabih* \\ University of Technology, Baghdad, Iraq
}

\begin{abstract}
A reinforced concrete column is classified as compression structural element mostly analyzed and designed due to the applied combinations of dead and live loading with other considered loadings. Industries of considerable or relatively great size, production and electrical utilities are very concerned about the presence of dynamic loads in their electrical power systems. This behavior provides current with different components that are multiples of the fundamental frequency of the system which are called harmonics. Reinforced concrete elements such as column must be checked for the strength capacity and the response due to applied harmonic loading after completed the static analysis and design. In present article evaluations of reinforced concrete columns under the effects of dynamic harmonic loadings are studied. The main parameters are the reinforcement ratio and harmonic ranged loadings. Finite elements approach was adopted to analyze the columns by ANSYS software and all models are simulated in three dimensions. The analysis results indicated that the square cross sections with that rectangular of the same cross sectional area are closed in performance against static and dynamic loadings.
\end{abstract}

\section{Introduction}

The response of reinforced concrete columns to dynamic loadings are very important to check out the static design of columns and to make the structural building safe in case of presence of dynamic loading. Many parameters that the reinforced concrete columns effecting in case of dynamic loading such as type of dynamic loading, amount of main reinforcements, tie reinforcements, type of concrete and the geometry of column. In 2004, Hideaki Nishida and Shigeki Unjoh [1], studied the performance of reinforced concrete columns (RCC) under the effects of dynamic loading such as earthquake. Different cross sections of columns were tested and the results showed the excitation of the column significant as nonlinear behavior of the column. In 2004, Siddik Sener et al [2], investigated the effects of RCC sizes due to axially loading. The square RCC with pin ended boundary condition was explored taking into account different slenderness ratios. The test results showed that stress at failure load decreased as the column size increased. In 2010, T. Yu et al [3], studied the non-uniform confinement of circular, square and other columns types using FEA by ABAQUS software. The analysis results indicated that close as compared with the test results by others researchers. In 2011, Zhishen $\mathrm{Wu}$ et al [4], explored the damaged that occurs in RCC under the effects of progressive seismic at the final stage. The test results be visible that the increased in progression of cracks due to reverse dynamic loads. In 2011, Hugo Rodrigues et al [5], looked out on the performance of RCC subjected to different histories as uniaxial and biaxial loading. The test results showed that a higher energy misspending in case of uniaxial than biaxial loading. In 2013, Libo Yan et al [6], investigated the performance RCC in presence of fiber reinforced polymer (FRP) under the effects of seismic loading. The test results showed that the presence of FRP enhancing the performance of RCC subjected to different loadings such as uniaxial and biaxial loading. In 2013, Yuanfeng Wang and Xiaoran Li [7], studied the hysteretic of RCC in case of presence of FRP subjected to cyclic loading. The nonlinear damping analysis using numerical method was adopted and the results indicated that the dynamic response of $\mathrm{RCC}$ more effectively reduced in case of taking into account the nonlinear damping analysis. In 2014, Ahmed Samir [8], studied the effects of blast loading on the RCC using finite elements approach FEA by ABAQUS software. Many parameters such as reinforcement ratios and charge weights were considered in study. The analysis results indicated that the residual displacement became more significant.

In present paper, the response of reinforced concrete

*Corresponding author: Saba.sabeeh@yahoo.com 
columns under the effects of dynamic harmonic loading with different parameters such as frequency ranges, longitudinal and transverse reinforcement will be

\section{Column strength capacity}

The reinforced concrete column is the compression member that reinforced by steel reinforcements to prevent buckling and to reducing the long term effects of creep in concrete. The tied column strength capacity based on the ACI - 318-2014 [9] as follow:

$$
\emptyset P_{\max }=0.8 \emptyset\left\{0.85 f_{G}^{\prime}\left(A_{g}-A_{g}\right)+f_{y} A_{g}\right\}
$$

In which, Pn, max is the nominal axial strength, $\phi$ safety factor equal to $(0.8)$, $\mathrm{f}$ 'is the compressive strength of concrete, $\mathrm{Ag}$ and $\mathrm{As}$ are the gross sectional area of concrete and area of main reinforcement steel respectively, fy is the yielding tensile strength of steel bar. The same material properties for the analyses were discussed and evaluate the strength capacity of RCC using FEA by ANSYS software

used as actual height and geometry of the columns. The main column is square with dimensions $(600 \times 600 \times 3000$ $\mathrm{mm}$ ) that transfer to circular column with diameter around to $(650 \mathrm{~mm})$ and rectangular with dimensions as $(450 \times 800 \mathrm{~mm})$. The main reinforcements adopt in present study based on the minimum requirements of ACI - 318 - 2014 [9] that (1.11\%) of gross sectional area of concrete. The main reinforcement for all cross sectional area is $(20 \phi 16)$ and the tied reinforced of column is $(\phi 10 @ 200 \mathrm{~mm})$ center to center along the columns. The design compressive strength of concrete is $(28 \mathrm{MPa})$ and the yield strength of reinforcement is (400 $\mathrm{MPa}$ ). The ultimate strength capacity of columns lists in Table (1). Figure (1) show the cross sectional area for all columns.

Table 1: Columns strength capacity

\begin{tabular}{|c|c|c|}
\hline Column mark & Geometry (mm) & $\begin{array}{c}\text { Column strength capacity } \\
(\mathbf{k N}) \text { Equation (1) }\end{array}$ \\
\hline Square Column (CS) & Square 600x600 & 6450 \\
\hline Rectangular Column (CR) & Rectangular 450x800 & 6450 \\
\hline Circular Column (CC) & Circular 650 & 6450 \\
\hline
\end{tabular}

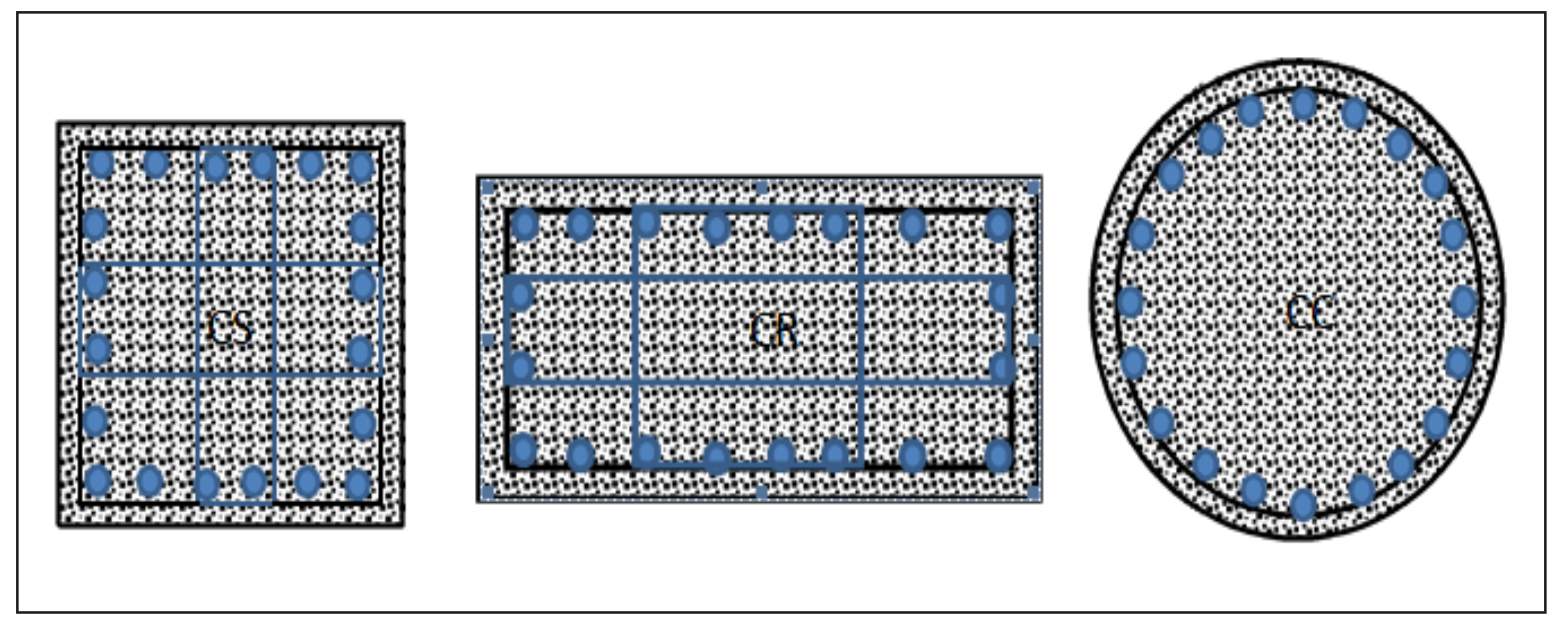

Fig. 1. The cross sectional area for all columns 


\section{Harmonic response analysis}

The applied continuing for an extended period or without interruption cyclic loading will produce a sustained cyclic response (harmonic response) in any type of structural system. Harmonic response analysis gives the ability to predict the sustained dynamic behavior of any structural elements such as the reinforced concrete columns, so that in this case enabling to verify whether or not the reinforced concrete columns design will successfully overcome resonance or other damaging effects of forced vibrations.The steady-state response of the reinforced concrete columns (RCC) subjected to

$[M\}\{\ddot{u}\}+[c]\{\dot{u}\}+[k]\{u\}=F_{O} \sin (\omega t) \ldots \ldots \ldots \ldots(2)$
sinusoidal with time and find out the response of the RCC at frequency range. The general equation of motion as follow: In which, $\mathrm{M}$ is mass matrix, c, is the damping matrix, $\mathrm{k}$, is the stiffness matrix, ü, u. and $u$ are the acceleration, velocity and displacement respectively. The solutions of the right hand side with amplitude force equal to the ultimate static loading and with frequency range as low (0-40) and medium (40-400).

proper material models, and boundary condition simulation for columns at the ends, convergence criteria and solution method are selected. The solver that used for static analysis was Newton - Raphson method and for dynamic analysis was Frontal solver with displacement convergence (5\%). The loading applied with sub-steps in case of static loading up to the ultimate loading. The dynamic analysis, first the modal analysis run to determine the frequencies that make the model resonance range for modal analysis. ANSYS [10] program is adopted to build all reinforced concrete columns that subjected to static with harmonic dynamic analysis. The solver for dynamic analysis that adopted in present study is sparse solver that takes into accounts the real and imaginary roots during solution of equations to find the unknown variables.

\section{Results}

The analysis results for reinforced concrete columns using finite elements approach by ANSYS are presented in Figures below. Figures (2) to (7) show that the longitudinal and horizontal displacements for all columns. Figures (8) to (10) represents the static analysis of models as longitudinal, horizontal displacements and longitudinal stress. Figures (11) to (16) show the performance of models under the

\section{Numerical analysis (finite elements approach)}

The numerical analysis is approximation approach as distinguished from discrete mathematics that used in present work as finite elements approach (FEA) by ANSYS [10] to simulate the reinforced concrete column models. The structural composed elements connected together at a finite number of nodes. Compatibility of displacement at any connections nodes between steel section and concrete will be satisfied due to the full bond between them. The full connections between the interior nodes of steel with the exterior nodes of concrete make the displacement same for the two materials at common nodes The numerical analysis adopted by FEA by ANSYS to simulate the composite columns relay on the suitable selection of elements type, so, SOLID65 [10], for concrete, SOLID185 for steel plate above the column that used to distributes the applied loading. In additions to the element types, number of elements,

applied harmonic loading as low and medium and presents the results of longitudinal, horizontal displacements and longitudinal stress.

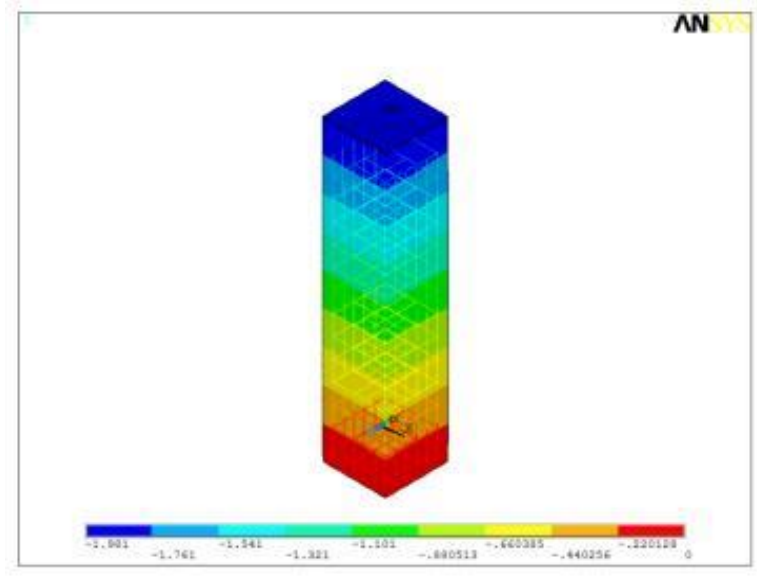

Fig. 2. Longitudinal displacement of CS (mm) 


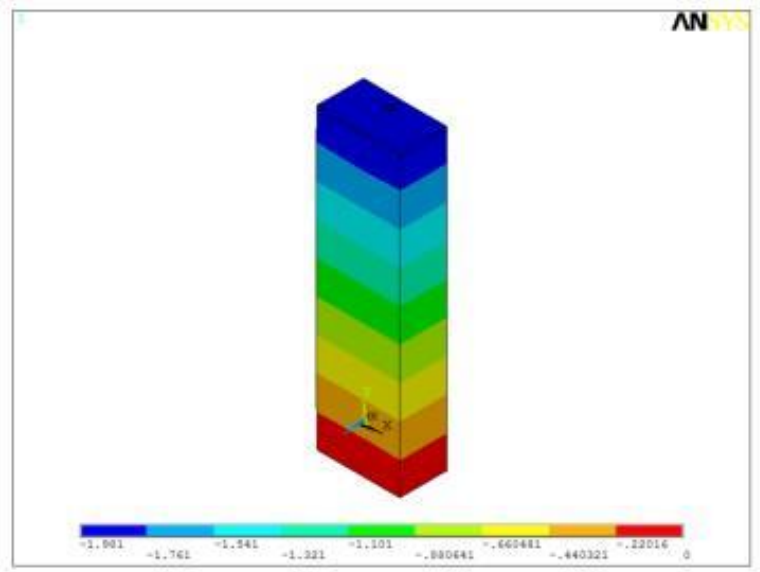

Figure 3: Longitudinal displacement of CR (mm)

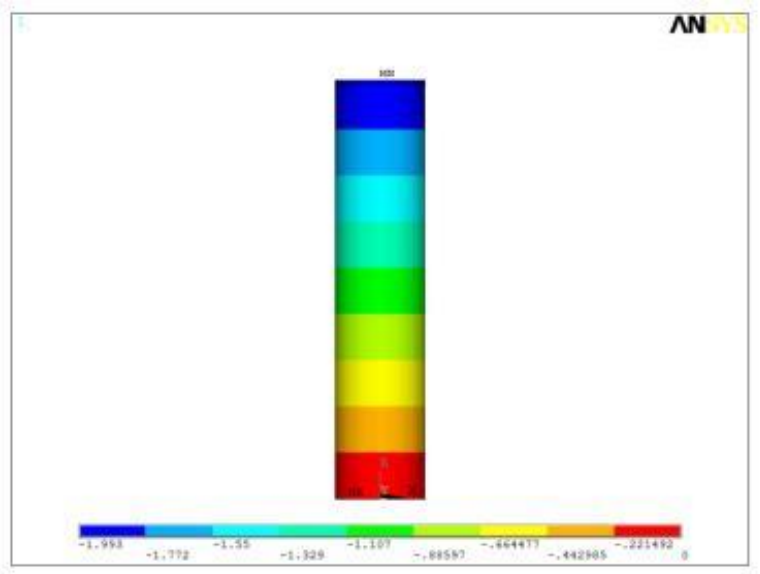

Figure 4: Longitudinal displacement of CC (mm)

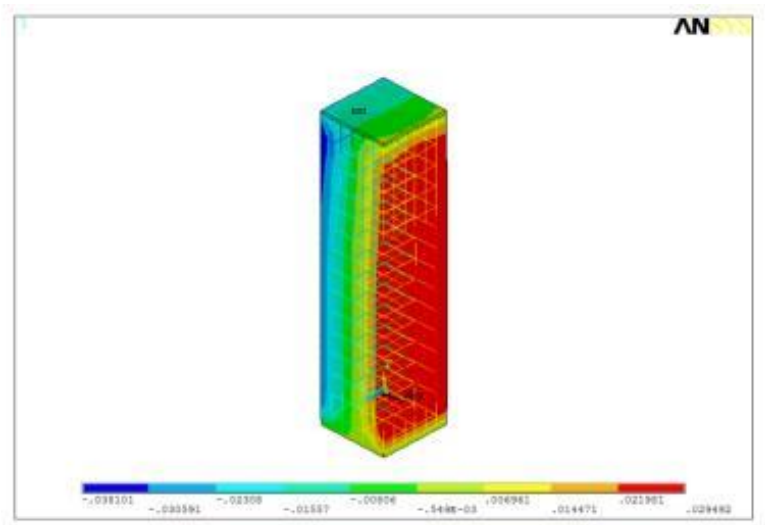

Figure 5: Horizontal displacement (ux) of CS (mm)

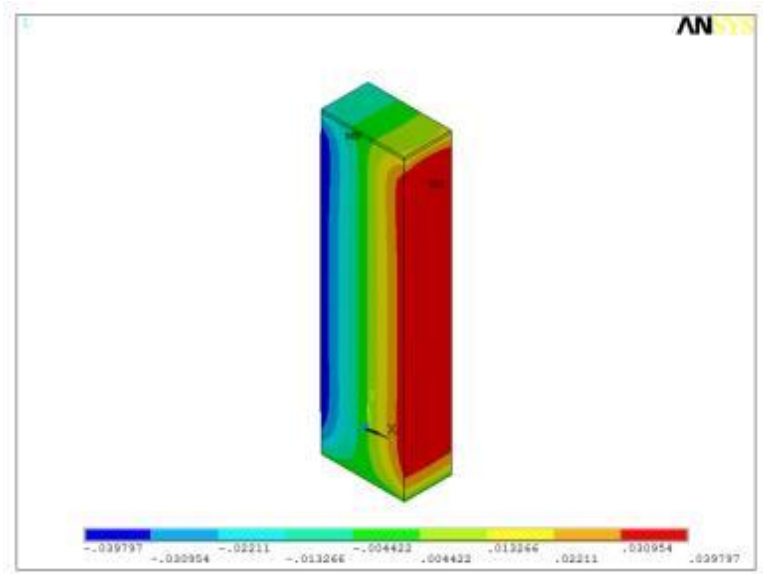

Figure (6): Horizontal displacement (ux) of CR $(\mathrm{mm})$

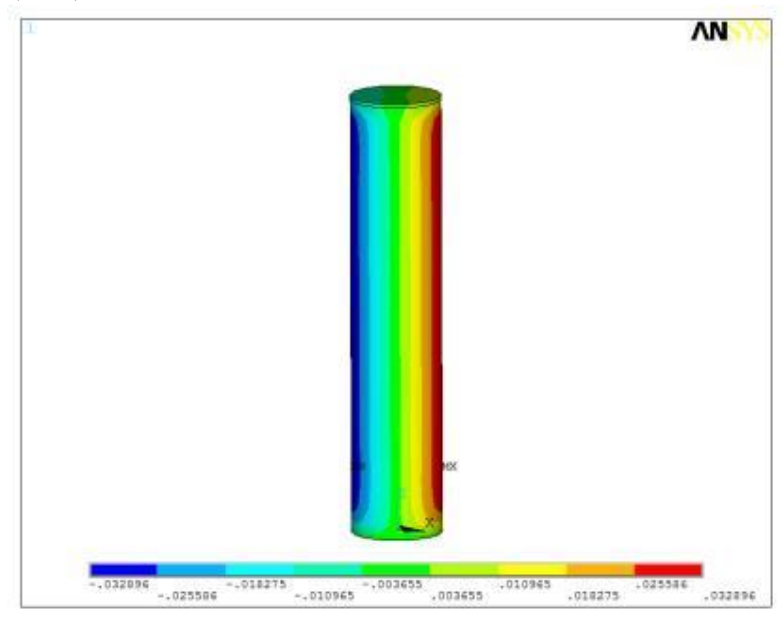

Figure 7: Horizontal displacement (ux) of CR (mm)

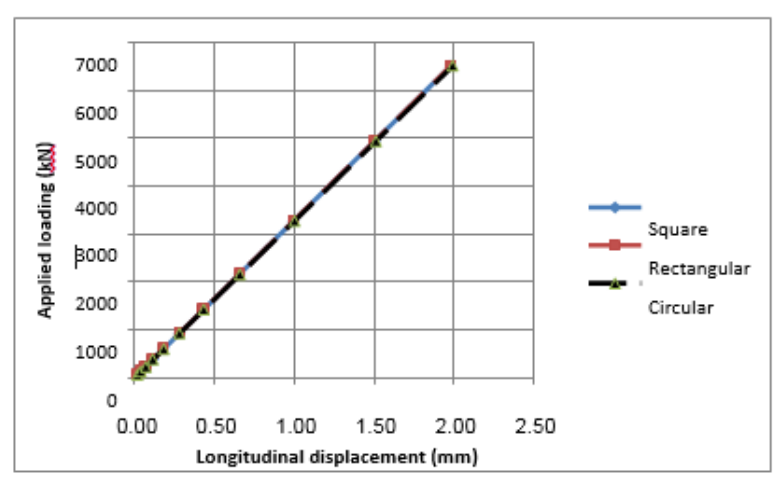

Figure 8: Applied loading vs. longitudinal displacement for all columns 


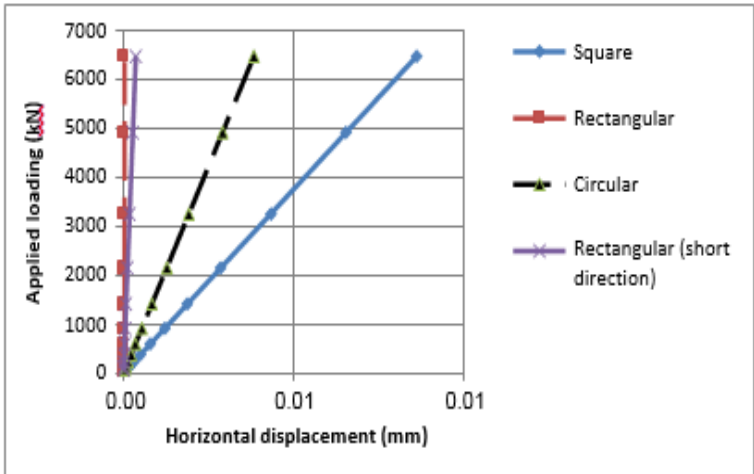

Figure 9: Applied loading vs. horizontal displacement for all columns

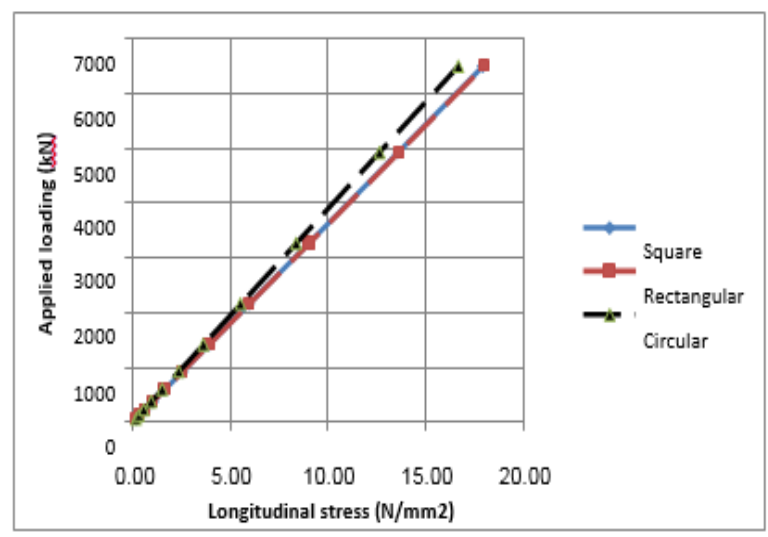

Figure 10: Applied loading vs longitudinal stress for all columns

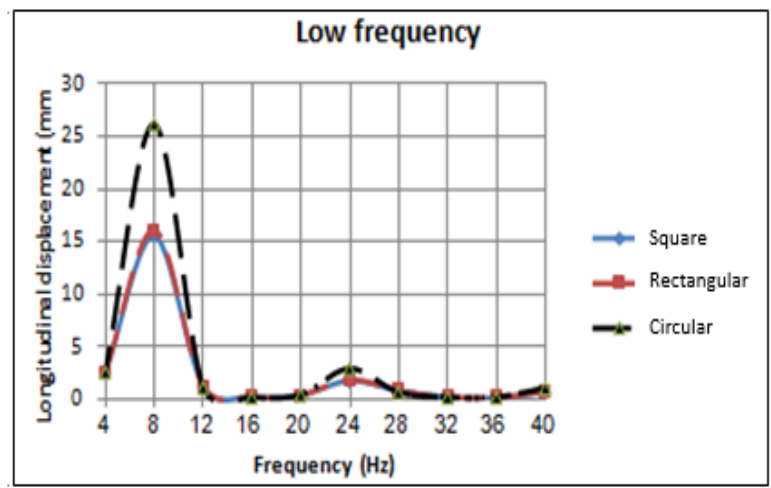

Figure 11: Longitudinal displacement for all columns vs. frequency (low)

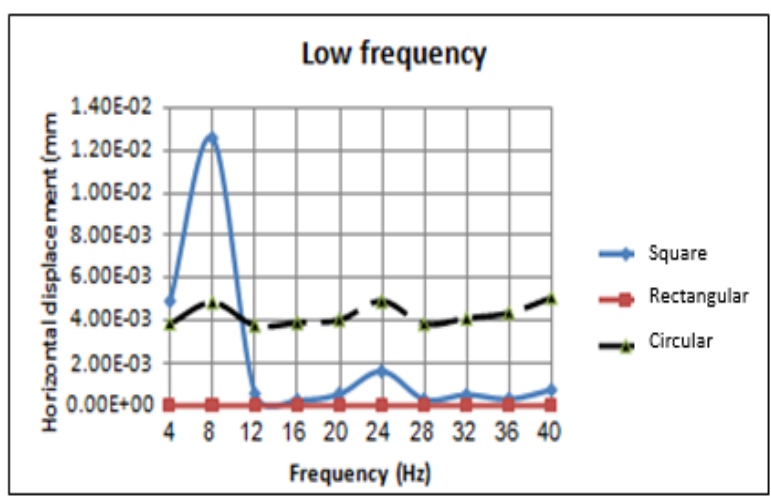

Figure 12: Horizontal displacement for all columns vs. frequency (low)

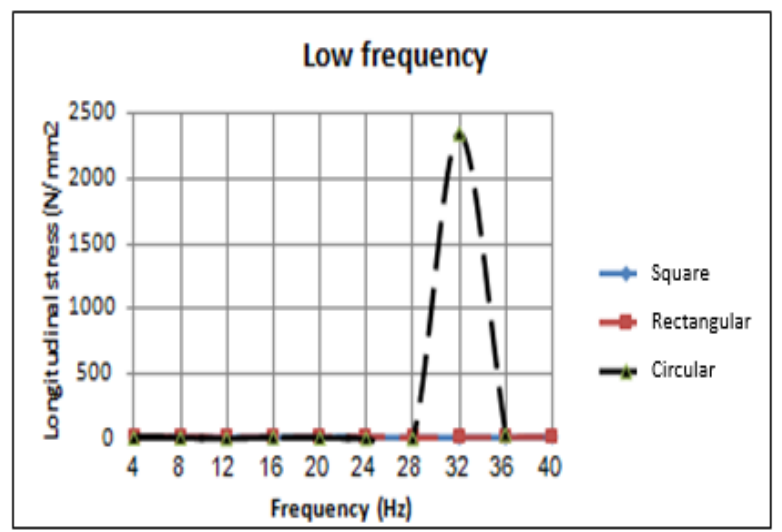

Figure (13): Longitudinal stress for all columns vs. frequency (low)

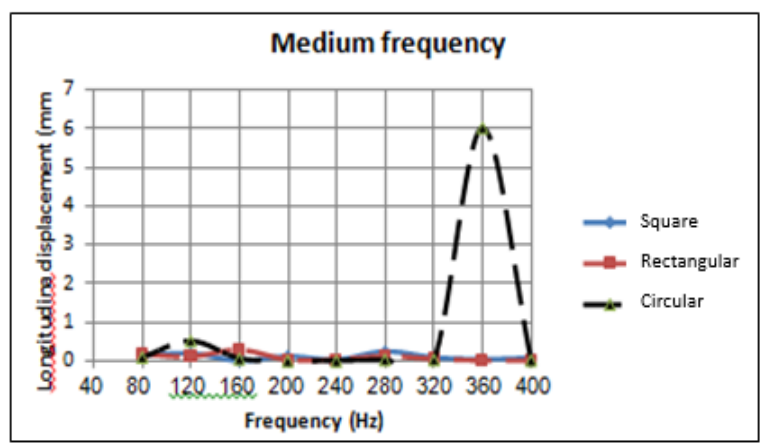

Figure 14: Longitudinal displacement for all columns vs. frequency (medium)

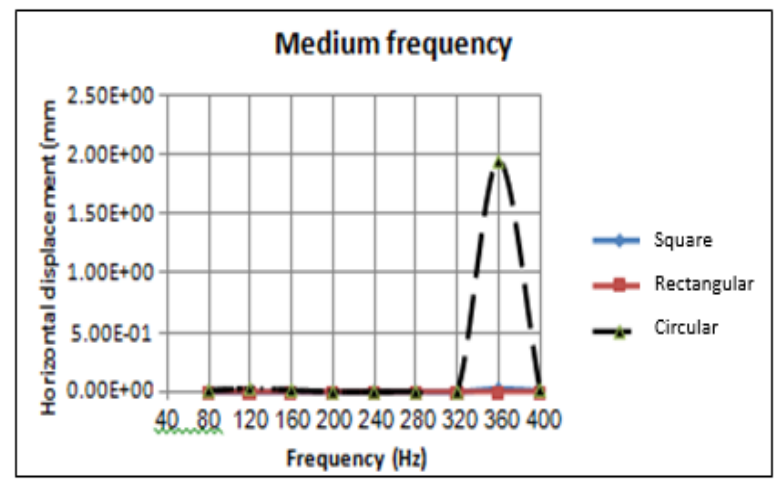

Figure 15: Horizontal displacement for all columns vs. frequency (medium)

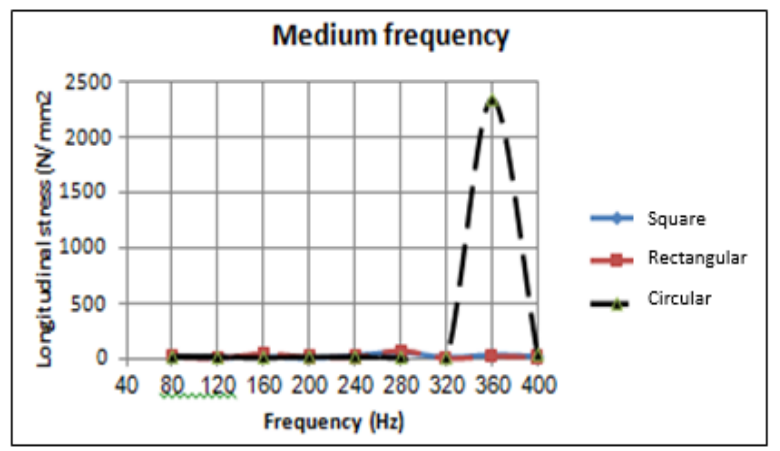

Figure 16: Longitudinal stress for all columns vs. frequency (medium) 


\section{Discussions}

Reinforced concrete columns were designed based on the ACI-318-2014 code under static loading as a square column first then make equivalent area to rectangular and circular columns with minimum steel reinforcements and then analyzed using finite elements approach and after that a harmonic analysis for all models are adopt to check out the full performance of the columns under dynamic loading Following points was concluded from study:

1.The static analysis results as longitudinal displacement approximately same for all columns cross section and closed with the axial displacement based on the strength of material principle that equal to $(2.10 \mathrm{~mm})$, for square $(1.98 \mathrm{~mm})$, for rectangular $(1.98 \mathrm{~mm})$ and for circular $(1.99 \mathrm{~mm})$ so that finite elements simulation deflection results by ANSYS software gives closed results.

2. The maximum horizontal displacement for square is $(0.0428 \mathrm{~mm})$, for rectangular is $(0.039$ and 0.0251 $\mathrm{mm})$ and for circular is $(0.0328 \mathrm{~mm})$. the rectangular column gave the lowest value of displacement as compared with other cross sections this is because of having smaller dimension in that direction

3. The longitudinal stress performance developed due to applied loading same for all sections because of the applied same load on the same area.

4. In case of low and medium frequency, the longitudinal displacement for circular section gave value more than other sections but at same frequency (8 and $360 \mathrm{~Hz}$ ) respectively, because of the stiffness of the circular column is less due to an approximate equivalent area rather than rectangular that gave same results for square section.

5. Stress developed due to the effects of applied harmonic loading are more than that produced due to static loading because the frequency range that applied on the models caused a stress-concentration at the point of maximum longitudinal displacement (points in which the axial displacement occur) that is mean at the point of applied loading. The frequency of circular section gave more stress concentration as compared with others cross section in case of low and medium frequency range.

6. The harmonic analysis must be taken into account to check out the reinforced concrete columns in case of presence of this type of loading because of the performance of the column is different under this type of loading.

7. Square and the equivalent rectangular cross section gave approximately same results and performance under static and dynamic loading.

8. No resonance will occur throughout the low and medium frequency ranges because of there are no infinite result.

\section{References}

1. H. NISHIDA, $13^{\text {th }}$ World Conference on Earthquake Engineering, August 1-6, Paper No. 576, (2004).

2. S.sener et al, J.Stru.Eng, volume $\mathbf{1 3 0}$ issue 4, april (2004).

3. T. Yu et al,ELSEVIER Engineering Structures, 680_691,PP.681-691, 32 (2010)

4. Z. wu et al, J. Earth. Tsun, Volume 05, Issue 02, June (2011).

5. H. Rodrigues et al, ELSEVIER Engineering Structures , 149-164 ,pp.150, 164, 35 (2012)

6. L. Yan et al, Australian Earthquake Engineering Society, Nov 15-17, Hobart, Tasmania ,pp.1-7.

7. Y. Wang Xiaoran Li, ELSEVIER Engineering Structures ,289-295 ,pp.289-294, 57 (2013)

8.A. Samir Eisa, International Journal of Civil and Structural Engineering, Volume 5 No 2, pp. 155$164 .$.

9. ACI Committee 318M-14, "Building Code Requirements for Structural Concrete and Commentary ", American Concrete Institute

10. ANSYS, Inc. Help Manual: is a UL registered ISO 9001:2000 Company. Version (16.2), USA, (2017). 